\title{
Volcanism on Io: the Post-Galileo View, and a Comparison with Earth
}

\author{
Ashley Gerard Davies \\ Jet Propulsion Laboratory-California Institute of Technology, 4800 Oak \\ Grove Drive, Pasadena, CA 91109, USA
}

\begin{abstract}
At the end of the Galileo mission in September 2003, and approximately three years after the Cassini Jovian flyby, it is an appropriate time to reflect on the recent advances made in Io science. Data have been analyzed and volcanic processes have begun to be quantified. This paper reviews these advances from a purely volcano-logical perspective, looking at particular volcanos of interest, and comparing styles of ionian activity with those seen on Earth.
\end{abstract}

\section{Recent Observations of Io}

During 2000-2002 Galileo made a number of close flybys of Io. While radiation caused many spacecraft and instrument problems, useful data were returned at resolutions as high as $6 \mathrm{~m} /$ pixel for the Solid State Imaging experiment (SSI). A number of analysis, made from Near-Infrared Mapping Spectrometer (NIMS) data analysis prior to the close encounters, were confirmed by these and other high-resolution data from NIMS and the Photo-Polarimeter Radiometer (PPR). Analysis of ground-based data, and the application of new technology in the form of Adaptive Optics ( $\mathrm{AO}$ ) has recently generated more insights to volcanic processes at individual sites: in the latter case, resolutions are obtained from Hawaii that are spatially equivalent to most NIMS data.

Io has a large $\mathrm{Fe}$ or FeS core, but no magnetic field. Visible aurora are seen, the result of charged particle interaction with volcanic gases. At Io, the Galileo spacecraft almost immediately detected high-temperature volcanism, evidence of lavas with temperatures indicative of silicate or even ultramafic composition. These high-temperature thermal sources were discovered all over the satellite, showing that silicate volcanism was the dominant, primary lava type, finally settling a question dating from the Voyager epoch. Sulphur may be an important secondary magma, and sulphur dioxide, ubiquitous on Io's surface, is an important volatile.

\section{The volcano tour: Pele, Prometheus and Loki}

Pele is the only ionian volcano that displays all the characteristics of an active lava lake, where the crust on the surface is being constantly disrupted by vigorous overturn, probably caused by the explosive de-gassing of lava. The sulphur-rich plume that results still forms the red deposits on the surface seen by Voyager. A night-time, multi-filter high-resolution SSI observation obtained in 2001 shows 
the distribution of temperatures topping out at about $1480 \mathrm{~K}$, in the basaltic composition range, confirming a previous NIMS analysis.

Prometheus has been described as Io's 'Old Faithful' as it was always seen to be producing a volcanic plume. However, unlike Pele, the plume at Prometheus appears to be generated as a result of gently effusive surface flows interacting with a $\mathrm{SO}_{2}$-rich surface, not exsolving from the lava. Accordingly, the plume deposits have moved with the newly emplaced flow field. Analysis of NIMS and SSI data show that over $6000 \mathrm{~km}^{2}$ of flows have been emplaced in the 17 years between Voyager and Galileo. The individual flow thicknesses at Prometheus (and other locations) are approximately 1-2 m thick. Additionally, it was recently discovered that volcanic activity at Prometheus was episodic in nature; every 7 to 9 months, there is a peak in thermal output at $5 \mu \mathrm{m}$. What is probably happening is that a sub-surface magma chamber is pressurizing as magma enters the chamber; the chamber eventually ruptures and magma migrates to the surface, and erupts. Pressure in the magma chamber decreases, and the eruption ends, the system seals, and the process starts again. Volumes of material erupted at Prometheus are greater by an order of magnitude than at the closest terrestrial analogue, Kilauea, Hawaii.

Loki is Io's power house, the most powerful volcano in the Solar System. Analysis of variation in 3.5-3.8 $\mu \mathrm{m}$ brightness revealed a periodicity at Loki of 540 days, interpreted as the foundering of the crust on an admittedly massive lava lake (over $150 \mathrm{~km}$ in diameter: lava lakes on Earth are typically $100 \mathrm{~m}$ across). Analysis of high-resolution NIMS data obtained in 2001 showed a surface distribution that indicated a diagonal resurfacing wave originating from the SW corner of the Patera, moving at a rate of about $1 \mathrm{~km}$ per day, consistent with PPR observations. Is Loki a huge lava lake, or is it being resurfaced by flows? It is not yet possible to say. If a lava lake, then Loki would be a window deep into the crust of Io.

\section{Thermal signatures and a comparison with terrestrial volcanism.}

Study of NIMS data, with model fits constrained by SSI and PPR data, have allowed different styles of volcanic activity to be identified on the basis of shape of infrared spectrum, mass and energy fluxes, and temporal behavior. Prometheus displays all the characteristics of a pahoehoe flow field; Pillan, a massive eruption accompanied by fire-fountaining, and open-channel, possibly turbulent flows, that were observed as they cooled over the following years.

Volcanism on Io is hotter, and produces more voluminous lavas than on Earth.

Volcanism on Earth produces about $18 \mathrm{~km}^{3}$ of lava per year; Io produces 480-540 $\mathrm{km}^{3}$ per year. Yet similar styles of volcanism look very much alike on these two planetary bodies. The energy and mass fluxes from pahoehoe flows, open-channel flows and active lava lakes look very similar on Io and Earth, which greatly aids interpretation of Io data.

This work was carried out at the Jet Propulsion Laboratory, California Institute of Technology, under contract to the National Aeronautics and Space Administration. The author is supported by a PG\&G grant. 